\title{
Experimental investigations on interaction of two drops by thermocapillary-buoyancy migration
}

\author{
Q. Kang *, L. Hu, C. Huang, H.L. Cui, L. Duan, W.R. Hu \\ National Microgravity Laboratory/CAS, Institute of Mechanics, Chinese Academy of Sciences, No. 15 BeiSiHuanXi Road, Beijing 100080, China
}

Received 5 July 2005; received in revised form 24 December 2005

Available online 23 March 2006

\begin{abstract}
Experiments were performed, in a terrestrial environment, to study the migration and interaction of two drops with different diameters in matrix liquid under temperature gradient field. Pure soybean oil and silicon oil were used as matrix liquid and the drop liquid, respectively. The information on the motions of two drops was recorded by CCD camera system in the experiments to analyze the trajectories and velocities of the drops. Our experiments showed that, upon two drops approaching each other, the influence of the larger drop on the motion of the smaller one became significant. Meanwhile the smaller drop had a little influence on the larger one all the time. The oscillation of migration velocities of both drops was observed as they were approaching. For a short period the smaller drop even moved backward when it became side by side with the larger one during the migration. Although our experimental results on the behavior of two drops are basically consistent with the theoretical predictions, there are also apparent differences.
\end{abstract}

(c) 2006 Elsevier Ltd. All rights reserved.

Keywords: Thermocapillary migration; Drop; Interaction; Oscillation

\section{Introduction}

A bubble or drop will move when placed in another fluid with temperature gradient. This motion happens as a consequence of the variation of interfacial tension with temperature. Such a phenomenon is already known as Marangoni migration problem. With the development of microgravity science, bubble dynamics and droplet dynamics became a hot point problem of research because this investigation is very important for basic research as well as for applications in reduced gravity environment, such as space material science, chemical engineering and so on.

Young et al. first investigated the thermocapillary migration of bubbles or drops with their linear YGB model when convective momentum and energy transport are both negligible [1]. The YGB model assumes that the applied temperature gradient is parallel to the direction of gravity

\footnotetext{
${ }^{*}$ Corresponding author. Tel.: +86 10 62626158; fax: +86 1062615524. E-mail address: kq@imech.ac.cn (Q. Kang).
}

and the migration velocity of a drop is the sum of two parts. That is

$$
\begin{aligned}
& V_{\mathrm{YGB}}=V_{\mathrm{m}}+V_{\mathrm{g}} \\
& V_{\mathrm{m}}=\frac{2\left(-\sigma_{\mathrm{T}}\right) R \kappa}{\left(2 \mu+3 \mu^{\prime}\right)\left(2 \kappa+\kappa^{\prime}\right)} \Gamma \\
& V_{\mathrm{g}}=\frac{2\left(\rho-\rho^{\prime}\right) g R^{2}\left(\mu+\mu^{\prime}\right)}{3 \mu\left(2 \mu+3 \mu^{\prime}\right)}
\end{aligned}
$$

where $V_{\mathrm{m}}$ is due to thermocapillary effect, and $V_{\mathrm{g}}$ is due to gravitational effect; $R$ is drop radius; $\Gamma$ is the temperature gradient of fluid field; $\sigma_{\mathrm{T}}$ is the temperature coefficient of interfacial tension; $\mu$ and $\mu^{\prime}, \kappa$ and $\kappa^{\prime}, \rho$ and $\rho^{\prime}$ are the dynamic viscosity, thermal diffusivity, and density of drop and matrix liquid, respectively.

The results from space experiments conducted by Hadland and Balasubramaniam et al. [2] and Xie [3,4] qualitatively confirm that the migration velocity trend of drop is accordant with the trend predicted by the YGB model, but the velocity are smaller than that given by the YGB model, 
and the velocity difference increases with the augment of drop radius.

In most applications, drops are not isolated. It is important to study the interactions of two or more drops. Up to date theoretical work was restricted to very small Reynold number and very small Marangoni number, so that all non-linear effects were omitted. Meyyappan [5] solved the axisymmetric thermocapillary migration of two gas bubbles in the quasi-steady state with bispherical coordinate. The result shows that two bubbles with the same size will migrate with same velocity regardless of thermal conductivity and interaction between two bubbles. The velocity is identical to that obtained from YGB model. Feuillebois [6] obtained an analytical solution. Bipolar coordinate is proper for analyzing axisymmetric problem but not for arbitrary ones. Subsequently, Meyyappan and Subramanian [7] solved the thermocapillary migration of two bubbles at arbitrary angle to the applied temperature gradient with zeroth-order reflection approximation. Then Anderson [8] extended the reflection solution to the first order and obtained the velocities of two arbitrarily oriented droplets up to terms of $\mathrm{O}\left(r_{12}^{-6}\right)$, where $r_{12}$ is the distance between the two drops. But all of these approximate solutions are invalid when the bubbles or drops move close enough. Acrivos et al. [9] estimated the average thermocapillary migration velocity of a cloud of identical bubbles. Keh and Chen [10] and Wei and Subramanian [11] used the boundary-collocation method to study the thermocapillary migration of a small number of bubbles. Sun and $\mathrm{Hu}$ $[12,13]$ used successive reflection technique to obtain analytical solution in 2002. The results indicate that the interaction between two bubbles has a significant influence on the migration of the smaller bubble. And when the larger bubble approaches to the smaller one and drives it aside, the smaller one's speed may decrease to zero and even move backwards. Fig. 1 gives one of their results, where $z_{1}, z_{2}$ and $y_{1}, y_{2}$ are the vertical and horizontal positions for small and large bubbles, respectively. The ratio $\lambda$ of larger radius $R_{2}$ to smaller radius $R_{1}$ is 10 . The initial center-to-center dimensionless distance $h_{0} / R_{1}$ is equal to 5.8 , where $h_{0}$ is initial center-to-center horizontal distance of two bubbles, and applied temperature gradient is $\Gamma=30 \mathrm{~K} / \mathrm{cm}$.

\section{Experimental apparatus and procedure}

This paper uses a liquid pair of matched densities to reduce the gravitational effect on drop migration. Immiscible pure soybean oil and $5 \mathrm{cSt}$ silicon oil are used as experimental media of the matrix liquid and drop, respectively. They are immiscible around room temperature. The pure soybean oil has the same density as $5 \mathrm{cSt}$ silicon oil at about $15^{\circ} \mathrm{C}$ and the difference of two liquid density is very small in the range of $15-90^{\circ} \mathrm{C}$. The difference of densities of two liquids is less than $0.02 \mathrm{~g} / \mathrm{cm}^{3}$, the relative difference is less than $2.4 \%$, and the variation of drop radius due to thermal expansion is less than $2 \%$. In this experiment, drops will migrate to hot wall under thermocapillary effect. Fig. 2 shows the variation of densities of $5 \mathrm{cSt}$ silicon oil and soybean oil plotted versus temperature.

As shown in Fig. 3, the rectangular test cavity is $70 \mathrm{~mm}$ high with the horizontal cross-section of $40 \times 40 \mathrm{~mm}^{2}$. The cell is composed of four optical glass walls and two aluminum blocks at the top and the bottom. A piece of electric heating film and a Peltier element are placed at the top

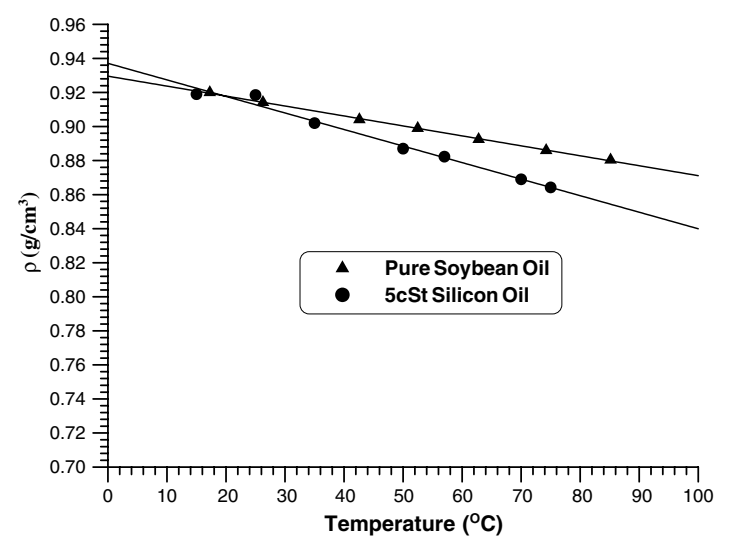

Fig. 2. Densities of $5 \mathrm{cSt}$ silicon oil and pure soybean oil plotted against temperature.

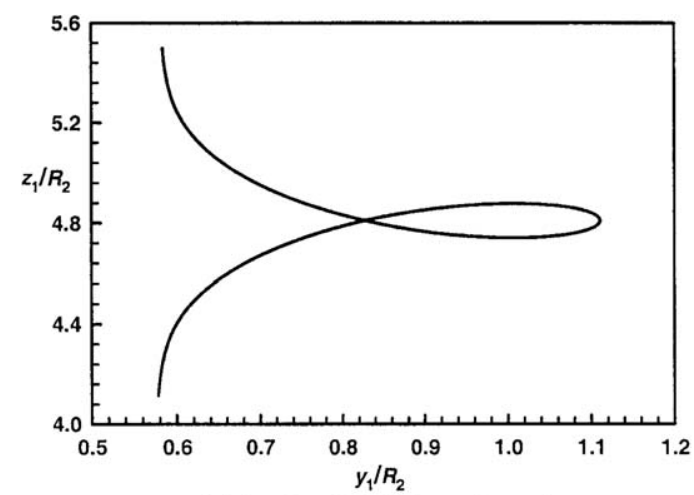

(a) Smaller bubble's trajectories

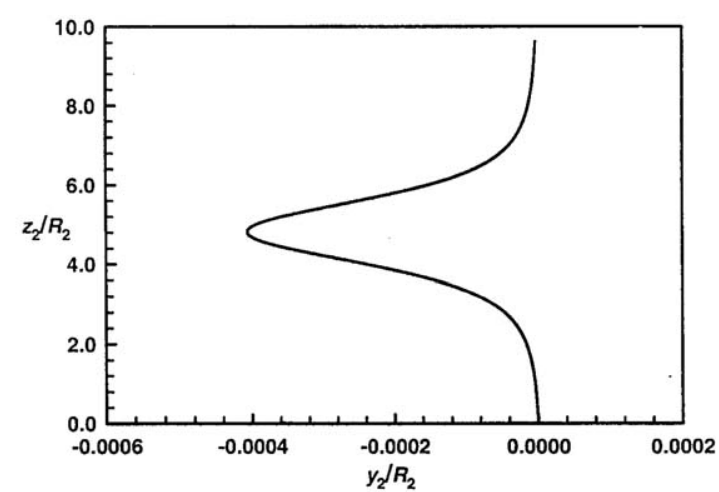

(b) Bigger bubble's trajectories

Fig. 1. Motions of two bubbles. 

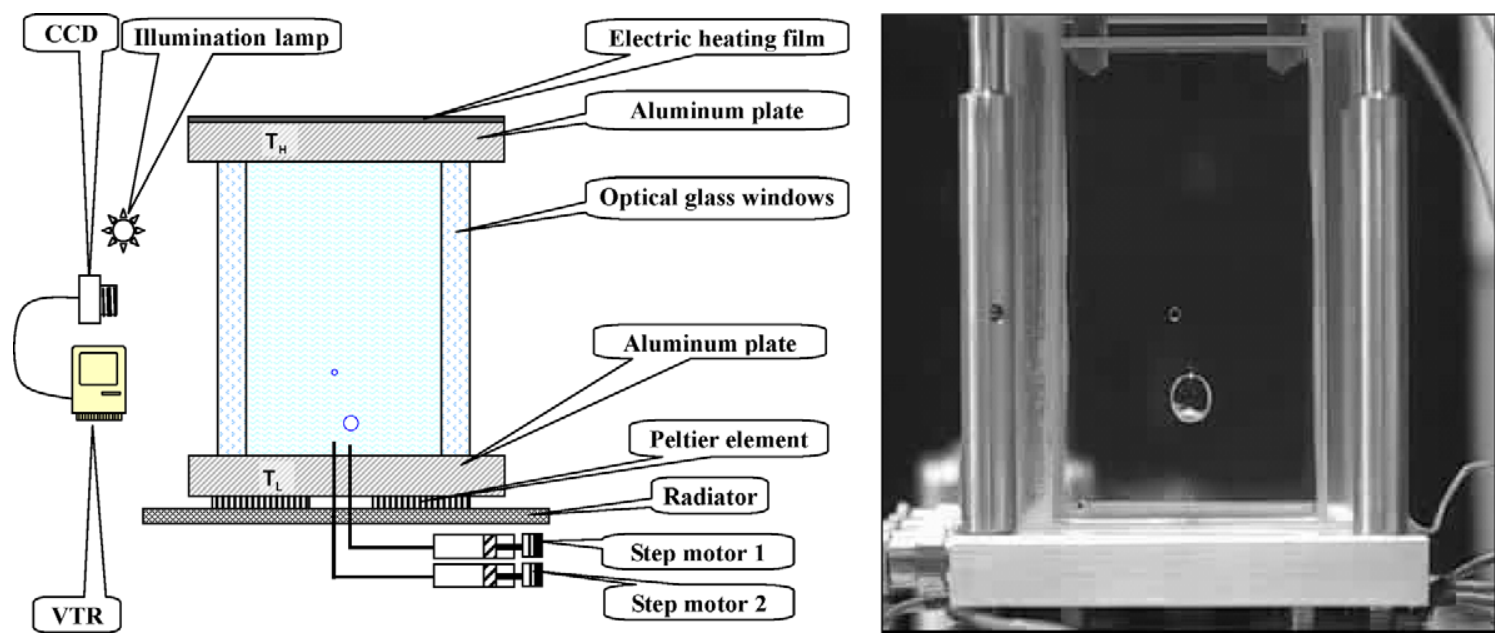

Fig. 3. Schematic representation of the experimental apparatus.

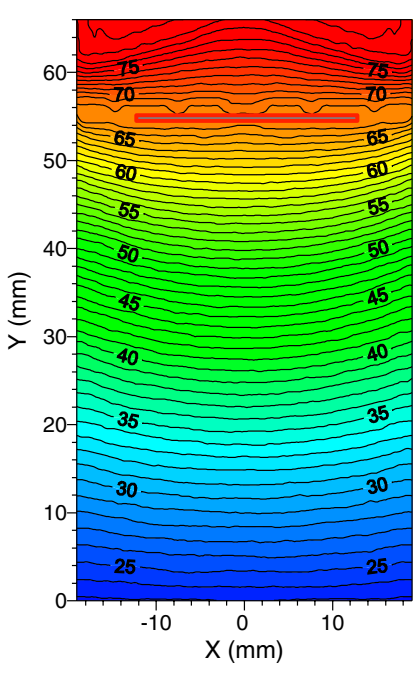

(a) Numerical simulation

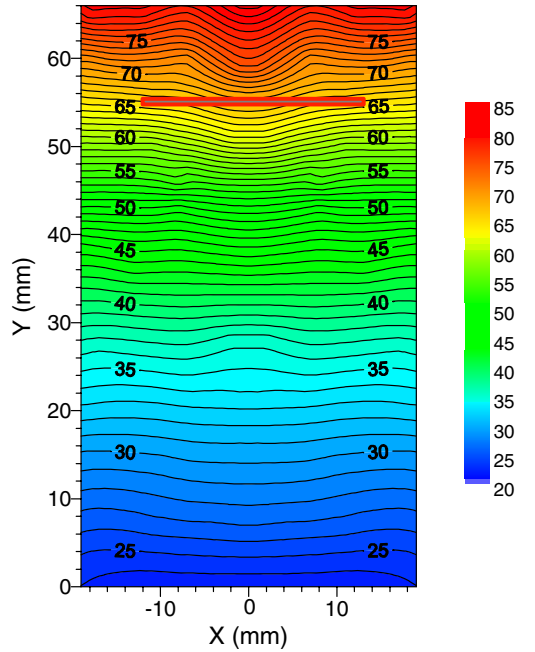

(b) Measurement with single thermocouple

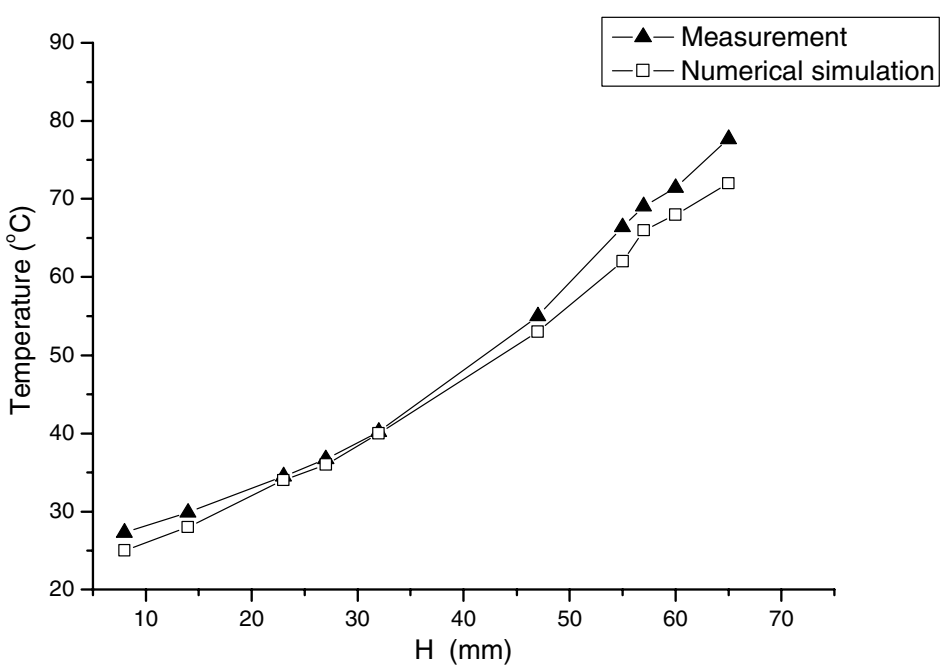

(c) Temperature at $\mathrm{X}=7 \mathrm{~mm}$

Fig. 4. Temperature field in the test cell. 
and the bottom surface, respectively, to maintain the temperature gradient. A grid plate is placed near the top to keep the uniformity of the temperature distribution and let drops staying at the top from disturbing temperature field.

In order to ensure a steady applied temperature gradient, a temperature controller of EUROTHERM 2132 (made in England) is used to measure and maintain a constant temperature at the bottom. And other temperature controller with a difference $\mathrm{T}$ type thermocouple is used to measure and control the temperature difference between the top and the bottom by controlling electric heating film at the top of cell.

In order to confirm the temperature field adequate to the experiment, numerical simulation is carried out by Fluent software. A 2D model is based on the experiment set-up mentioned in the foregoing statement. The size of the cell is $70 \mathrm{~mm} \times 40 \mathrm{~mm}$. The thermal conductivity of optical glass and aluminum is $1.207 \mathrm{~W} / \mathrm{m} \mathrm{K}$ and $203 \mathrm{~W} / \mathrm{m} \mathrm{K}$. Convective heat transfer boundary condition is specified at the glass wall and a fixed temperature condition is applied at the aluminum block. Fig. 4(a) shows the numerical simulation result of the temperature distributions in the cell, and Fig. 4(b) shows the measured result with a scanned thermocouple, when the temperature at the bottom is $20^{\circ} \mathrm{C}$ and the temperature difference between top and bottom is $60{ }^{\circ} \mathrm{C}$. Fig. 4(c) shows the difference of temperature at $X=7 \mathrm{~mm}$ between numerical simulation and measurement. The mean of the difference is $2.41^{\circ} \mathrm{C}$. The results show that the measurement is identical to the numerical simulations and there is enough uniformity temperature gradient in the middle part of the cell. Besides, before the experiments of drop migration were made, PIV technique was used to learn convective situation in the test cell. The experimental result showed that convection really does not exist in the fluid field.

In order to control the radius of drop injected accurately, a small diameter of cylinders (made by FESTO in Germany) and Stepper-Mike Actuators (made by PI in Germany) are used to control the volume of drop injected. A CCD camera, the illumination lamp and VTR consist of the recording system of drop track.

\section{Experimental results}

In the experiment, after a steady temperature gradient parallel to the direction of gravity is established, a small drop is injected first. When the small drop moves near to the middle of the cell, a large drop will be injected. The large trailing drop will chase and pass the small leading drop, because the large drop migrates faster than the small one under an applied temperature gradient according to the YGB model. Moving drops are monitored and recorded on-line by the recording system, so drop size, trajectories and velocities can be obtained by analyzing the image. Fig. 5 shows typical trajectories of two drops.

When the larger drop overtakes the smaller one, the larger drop only has a small horizontal movement, but the

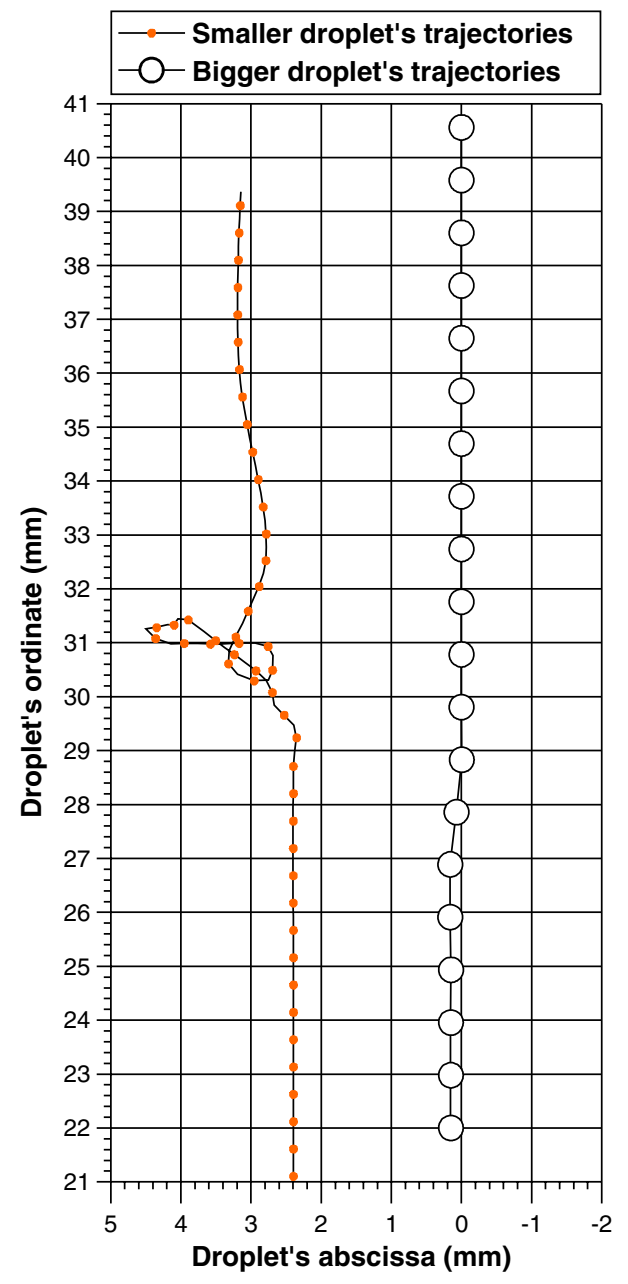

Fig. 5. Droplet's trajectories.

smaller is greatly affected. The smaller does not only move horizontally much more than the larger, but also move backwards. Its trajectory looks like an oblique number ' 8 ' that is consistent with the results given by Sun and $\mathrm{Hu}$ $[12,13]$.

The major properties of the liquid system used in the experiment are listed in Tables 1 and 2.

The temperature coefficient of interfacial tension $\sigma_{\mathrm{T}}=$ $-0.0088 \mathrm{dyn} / \mathrm{cm} \mathrm{K}$. Applied temperature gradient $\Gamma=$ $10.6 \mathrm{~K} / \mathrm{cm}$. The ratio of radius $\lambda=3.5$, initial center-tocenter dimensionless distance $h_{0} / R_{1}=2.5$. Figs. 6 and 7 show the dimensionless velocity $V / V_{\mathrm{YGB}}$ against time where $V$ is the real velocity of drop, $V_{\mathrm{YGB}}$ is the velocity obtained from the YGB model.

From Figs. 6 and 7, while the large drop chases after the small drop, the interaction between the two drops can be neglected, when one drop is far from the other as $h / R_{2}>2$, where $h$ is vertical distance of two drops. And two drops migrate with their individual steady velocities that much less than $V_{\mathrm{YGB}}$. When one drop is close enough to the other, the vertical velocities of the two drops all appear with great oscillations, and the max speed of drops are even close to or more than $V_{\mathrm{YGB}}$. Especially, the small 
Table 1

Physical property parameters of pure soybean oil

\begin{tabular}{|c|c|c|c|c|c|c|c|}
\hline \multirow[t]{2}{*}{ Properties } & \multicolumn{7}{|c|}{ Temperature $\left({ }^{\circ} \mathrm{C}\right)$} \\
\hline & 17.25 & 26.26 & 42.60 & 52.48 & 62.80 & 74.24 & 85.15 \\
\hline$\rho\left(\mathrm{g} / \mathrm{cm}^{3}\right)$ & 0.9200 & 0.9142 & 0.9041 & 0.8990 & 0.8925 & 0.8860 & 0.8804 \\
\hline$\kappa(\mathrm{mW} / \mathrm{mg} \mathrm{K})$ & 176.1 & 175.3 & 173.7 & 172.8 & 171.8 & 170.8 & 169.7 \\
\hline$\mu\left(c p=10^{-2}\right.$ dyn $\left.\mathrm{s} / \mathrm{cm}^{2}\right)$ & 86.3 & 62.6 & 31.6 & 22.5 & 17.3 & 12.5 & 10.9 \\
\hline
\end{tabular}

Table 2

Physical property parameters of $5 \mathrm{cSt}$ silicon oil

\begin{tabular}{|c|c|c|c|c|c|c|c|}
\hline \multirow[t]{2}{*}{ Properties } & \multicolumn{7}{|c|}{ Temperature $\left({ }^{\circ} \mathrm{C}\right)$} \\
\hline & 15.0 & 25.0 & 35.0 & 50.0 & 57.0 & 70.0 & 75.0 \\
\hline$\rho\left(\mathrm{g} / \mathrm{cm}^{3}\right)$ & 0.9190 & 0.9185 & 0.9020 & 0.8870 & 0.8823 & 0.8690 & 0.86424 \\
\hline$\kappa(\mathrm{mW} / \mathrm{mg} \mathrm{K})$ & 112.1 & 111.0 & 109.9 & 108.3 & 107.6 & 105.4 & 104.5 \\
\hline$\mu\left(c p=10^{-2}\right.$ dyn $\left.\mathrm{s} / \mathrm{cm}^{2}\right)$ & 5.09 & 4.27 & 3.61 & 2.86 & 2.67 & 2.17 & 2.01 \\
\hline
\end{tabular}

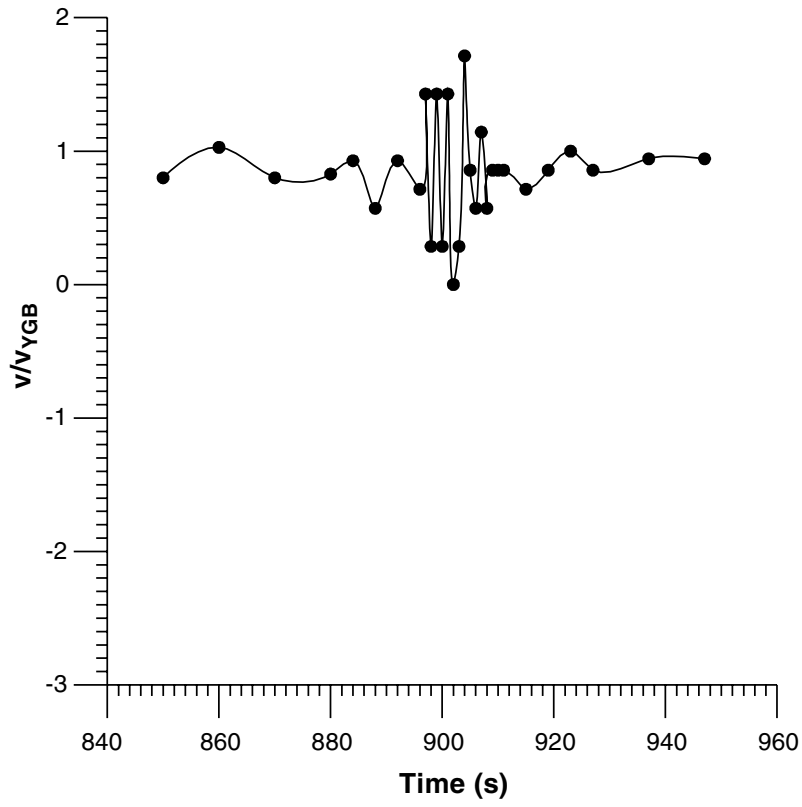

Fig. 6. Vertical velocity of bigger droplet.

drop moves backwards with the velocity of twice $V_{\mathrm{YGB}}$, and the large one decreases its speed close to zero.

In the experiment, the maximum $R e$ and $M a$ are 0.028 and 5.68, respectively, for the small drop, and 0.146 and 29.09 for the large one. The results show that the drop migrations in the case of small $\operatorname{Re}<1$ and intermediate $M a$ of $\mathrm{O}\left(10^{1}\right)$, so non-linear effects, especially caused by thermocapillary effect, should be considered. This is an important reason why there are some differences between the experiment result and the simulation result by Sun and $\mathrm{Hu}[12,13]$. On the other hand, buoyancy effect cannot be ignored in the extent of experiment temperature because of the difference of thermal dilatability between two liquids. At $70{ }^{\circ} \mathrm{C}$ thermocapillary migration velocity of the small drop $V_{\mathrm{m} 1}=0.1497 \mathrm{~mm} / \mathrm{s}$ and buoyancy migration velocity

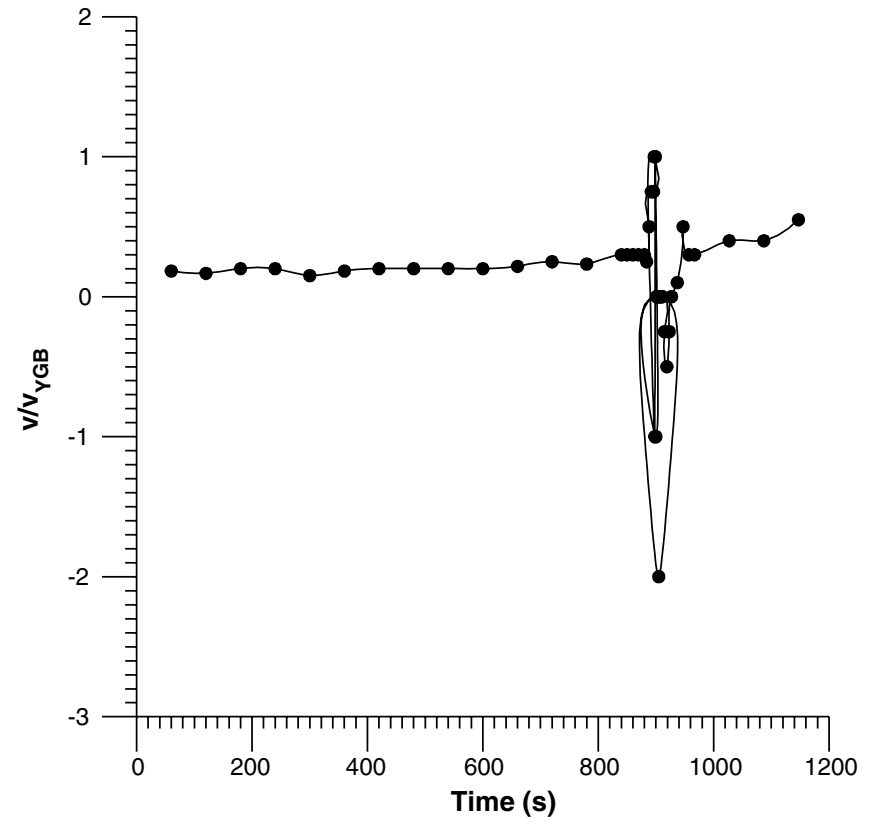

Fig. 7. Vertical velocity of smaller droplet.

of the small drop $V_{\mathrm{g} 1}=0.0230 \mathrm{~mm} / \mathrm{s}$, the ratio of the two velocities $V_{\mathrm{m} 1} / V_{\mathrm{g} 1}=6.5$ was obtained from the $\mathrm{YGB}$ model; and that for the large drop, $V_{\mathrm{m} 2}=0.5240 \mathrm{~mm} / \mathrm{s}$, $V_{\mathrm{g} 2}=0.2815 \mathrm{~mm} / \mathrm{s}, V_{\mathrm{m} 2} / V_{\mathrm{g} 2}=1.86$. The results show that buoyancy effect increases with drop size. This in accord with Eq. (3).

\section{Conclusion}

The presented results on the motion and interaction of two drops demonstrate that the interaction between the two drops can be neglected, when one drop was far from the other $\left(h / R_{2}>2\right)$, and that the effect of the larger drop on the motion of the smaller one becomes significant with 
the two drops approaching each other, while the effect of the smaller one on the larger remains weak. It is found that both migration velocities oscillate during the approaching process. Even the smaller one moves backwards in a short period when two drops migrate side by side so that the trajectory of smaller one looks like a number ' 8 '. They display the congruence and difference between the experimental results and numerical results.

\section{Acknowledgements}

This work was supported by the National Natural Science Foundation of China (10432060) and Knowledge Innovation Program of Chinese Academy of Sciences (KJCX2-SW-L05, KSCX2-SW-322).

\section{References}

[1] N.O. Young, J.S. Goldstein, M.J. Block, The motion of bubbles in a vertical temperature gradient, J. Fluid Mech. 6 (1959) 350-356.

[2] P.H. Hadland, R. Balasubramaniam, G. Wozniak, R.S. Subramanian, Thermocapillary migration of bubbles and drops at moderate values of the Marangoni number in reduced gravity, Exp. Fluids 26 (1999) 240-248.
[3] J.C. Xie, H. Lin, J.H. Han, X.Q. Dong, W.R. Hu, A. Hirata, M. Sakurai, Experimental investigation of thermocapillary migration of isolated drops, Adv. Space Res. 24 (10) (1999) 1409-1415.

[4] J.C. Xie, H. Lin, P. Zhang, F. Liu, W.R. Hu, Space experiment on thermocapillary drop migration at large Marangoni, in: 54th International Astronautical Congress, Bremen, Germany, IAC-03-J.3.03, 2003.

[5] M. Meyyappan, W.R. Wilcox, R.S. Subramanian, The slow axisymmetric motion of two bubbles in a thermal gradient, J. Colloid Interf. Sci. 94 (1983) 243-257.

[6] F. Feuillebois, Thermocapillary migration of two equal bubbles parallel to their line of centers, J. Colloid Interf. Sci. 131 (1989) $267-$ 274.

[7] M. Meyyappan, R.S. Subramanian, The thermocapillary motion of two bubbles oriented arbitrarily relative to a thermal gradient, J. Colloid Interf. Sci. 97 (1984) 291-294.

[8] J.L. Anderson, Droplet interactions in thermocapillary motion, Int. J. Multiphase Flow 11 (1985) 813-824.

[9] A. Acrivos, D.J. Jeffrey, D.A. Saville, Particle migration in suspensions by thermocapillary or electrophoretic motion, J. Fluid Mech. 212 (1990) 95-110.

[10] H.J. Keh, L.S. Chen, Droplet interactions in axisymmetric thermocapillary motion, J. Colloid Interf. Sci. 151 (1992) 1-16.

[11] H. Wei, R.S. Subramanian, Thermocapillary migration of a small chain of bubbles, Phys. Fluids A5 (7) (1993) 1583-1595.

[12] R. Sun, W.R. Hu, The thermocapillary migration of two bubbles in microgravity environment, J. Colloid Interf. Sci. 255 (2002) 375-381.

[13] R. Sun, W.R. Hu, Planar thermocapillary migration of two bubbles in microgravity environment, Phys. Fluids A 15 (10) (2003) 3015-3027. 